\title{
Effect of erythropoietin on angiogenic potential of dental pulp cells
}

\author{
QIMEI GONG ${ }^{1 *}$, JUNYU ZENG $^{2 *}$, XUFANG ZHANG ${ }^{1}$, YIHUA HUANG $^{1}$, \\ CHANCHAN CHEN $^{3}$, JINGJING QUAN ${ }^{1}$ and JUNQI LING ${ }^{1}$
}

\author{
${ }^{1}$ Department of Operative Dentistry and Endodontics, Hospital of Stomatology, Guanghua School of Stomatology, \\ Sun Yat-sen University and Guangdong Provincial Key Laboratory of Stomatology, Guangzhou, Guangdong 510055; \\ ${ }^{2}$ Stomatological Hospital, Southern Medical University, Guangzhou, Guangdong 510515; ${ }^{3}$ Department of Stomatology, \\ Shenzhen Children's Hospital, Shenzhen, Guangdong 518038, P.R. China
}

Received February 8, 2021; Accepted June 21, 2021

DOI: $10.3892 / \mathrm{etm} .2021 .10513$

\begin{abstract}
Erythropoietin (EPO) is a 34-kDa glycoprotein that possesses the potential for angiogenesis, as well as antiinflammatory and anti-apoptotic properties. The present study aimed to examine the effect of EPO on the angiogenesis of dental pulp cells (DPCs) and to explore the underlying mechanisms of these effects. It was demonstrated that EPO not only promoted DPCs proliferation but also induced angiogenesis of DPCs in a paracrine fashion. EPO enhanced the angiogenic capacity by stimulating DPCs to secrete a series of angiogenic cytokines. ELISA confirmed that high concentrations of EPO increased the production of MMP-3 and angiopoietin-1 but decreased the secretion of IL- 6 . Furthermore, EPO activated the ERK1/2 and p38 signaling pathways in DPCs, while inhibition of these pathways diminished the angiogenesis capacity of DPCs. The present study suggested that EPO may have an important role in the repair and regeneration of dental pulp.
\end{abstract}

\section{Introduction}

The dental pulp is vulnerable to infection resulting from dental caries and injuries. Most dental pulp infections are irreversible due to ischemia induced by anatomic factors, since the dental pulp is surrounded by rigid dentin walls and has limited collateral circulation. The survival of inflamed vital pulp is closely associated with the process of angiogenesis (1). Angiogenesis

Correspondence to: Professor Junqi Ling, Department of Operative Dentistry and Endodontics, Hospital of Stomatology, Guanghua School of Stomatology, Sun Yat-sen University and Guangdong Provincial Key Laboratory of Stomatology, 56 West Lingyuan Road, Guangzhou, Guangdong 510055, P.R. China

E-mail: lingjq@mail.sysu.edu.cn

\section{${ }^{*}$ Contributed equally}

Key words: erythropoietin, dental pulp cells, angiogenesis, proliferation, pulp regeneration provides blood supply, oxygen and nutrients, which are indispensable during dental pulp repair and regeneration.

Angiogenesis is a multi-sequential-step procedure involving the interaction between endothelial or stem cells, extracellular matrix components and mediation of various pro-angiogenic and anti-angiogenic factors (2). Dental pulp cells (DPCs) are a heterogeneous population of dental pulp stem cells (DPSCs) and other progenitors that possess mesenchymal stem cell (MSC)-like properties (3). DPSCs have been reported to induce angiogenesis in a paracrine fashion by secreting angiogenic molecules (4). Under extreme circumstances, such as serum deprivation, the secretome of DPSCs was observed to enhance the pulp repair ability, but this effect was not obvious under normal serum conditions (5). Furthermore, a recent study indicated that DPSC-conditioned medium (CM) accelerated the adhesion, proliferation, migration and tubulogenesis of human umbilical vein endothelial cells (HUVECs) (6). Accumulating evidence suggests that DPCs or DPSC-derived CM has angiogenic potential (7). However, the mechanisms underlying the angiogenesis of dental pulp have remained to be completely elucidated.

Erythropoietin (EPO) is mainly synthesized in the fetal liver and adult kidney and was initially highlighted for its action on the hematopoietic system (8). Extensive studies indicated that EPO and EPO receptor (EPOR) may be detected in a wide range of nonhematopoietic tissues and cells and have an important role in angiogenesis, anti-inflammation and anti-apoptosis (9-11). For instance, EPO enhanced the angiogenic capacity in a model of cerebral unilateral hypoxia-ischemia, bone repair and burn injury (12-14). Previous studies suggested that EPO was expressed in inflamed dental pulp but rarely detected in healthy dental pulp. EPOR was detected in both healthy and inflamed dental pulp. EPO and EPOR expressions in DPCs were increased under hypoxia (15). Given that dental pulp is susceptible to ischemia conditions (hypoxia and serum deprivation) when exposed to trauma or inflammation, it was hypothesized that EPO may have a role in enhancing the angiogenic capacity of DPCs.

MAPKs regulate cellular proliferation, development, differentiation and apoptosis through cascade amplification, and respond to a variety of extracellular stimuli, such as inflammation, physical and chemical stress. The activation 
of MAPK pathways is involved in angiogenesis by regulating the secretion of cytokines. Furthermore, it was revealed that EPO binding to EPOR activates pathways downstream of MAPK (16). However, it is unknown whether MAPK is involved in the angiogenesis of EPO-induced DPCs.

In the present study, the effects of EPO on the proliferation and angiogenesis of DPCs were studied. To explore how EPO works on DPCs, the cytokine profile of DPCs in response to EPO was investigated by using an angiogenic cytokine array under serum deprivation conditions. Furthermore, the involvement of signaling pathways was investigated by detecting proteins of the MAPK signaling pathway. The present study supplements the previously known angiogenesis effect of EPO on DPCs and may also provide novel perspectives in pulp repair and regeneration.

\section{Materials and methods}

Isolation and culture of DPCs. Integral premolars and impacted third molars were collected from young patients (10 males and 8 females; $18-25$ years of age; recruited between January 2014 and January 2018) who underwent surgical orthodontic treatment at the Hospital of Stomatology of Sun Yat-sen University (Guangzhou, China). None of the patients had clinically significant medical history, nor were they receiving medication. Ethics approval for the present study was obtained from the Research Ethics Committee of Hospital of Stomatology, Guanghua School of Stomatology, Sun Yat-sen University and informed consent was obtained from each patient prior to obtainment of the samples. The DPCs were isolated and cultured as previously reported (15). DPCs cultured from the third to sixth passages were used in the subsequent experiments.

Cell counting Kit-8 (CCK-8) assay. DPCs were seeded into 96-well plates at a density of $4 \times 10^{3}$ cells/well. After overnight incubation, different concentrations of EPO $(0.1,1,5,10,20$ and $40 \mathrm{U} / \mathrm{ml} ; \mathrm{R} \& D$ Systems, Inc.) were added to each well of cells in the treatment groups, while cells in serum-free DMEM only served as the control group. After being cultured for 1, 2 or 3 days, CCK-8 stain (Dojindo Molecular Technologies, Inc.) was added to each well followed by incubation at $37^{\circ} \mathrm{C}$ in the dark for $3 \mathrm{~h}$, according to the manufacturer's protocol. The optical density was measured at $450 \mathrm{~nm}$ using a microplate reader (BioTek). The results were calculated as the mean of three replicates of three experiments under the same conditions.

Tube formation assay on matrigel ${ }^{\circledR}$. CM was collected according to the following procedure: DPCs were cultured in $25-\mathrm{cm}^{2}$ flasks at $90 \%$ confluence in $1 \mathrm{ml}$ serum-free DMEM with $40 \mathrm{U} / \mathrm{ml}$ EPO for $24 \mathrm{~h}$. Subsequently, the CM was collected, centrifuged for $20 \mathrm{~min}$ at $10^{4} \mathrm{x} \mathrm{g}$ at $4^{\circ} \mathrm{C}$ and used in the subsequent experiments. HUVECs (cat. no. 8000; ScienCell Research Laboratories, Inc.) were cultured in complete endothelial cell medium (ScienCell Research Laboratories, Inc.). For the tube formation assay, HUVECs $\left(4 \times 10^{4}\right.$ cells per well) were seeded onto each well precoated with Matrigel ${ }^{\circledR}(350 \mu \mathrm{l} /$ well; BD Biosciences) in 48-well plates. CM with or without $40 \mathrm{U} / \mathrm{ml}$ EPO neutralization antibody (cat. no. AB-286-NA; R\&D Systems, Inc.) was added to HUVECs after plating and cultured for $4 \mathrm{~h}$. Tube formation images were obtained using an inverted microscope (Zeiss AG) and 3 fields per well were randomly selected for analysis of tube lengths branch points and amounts of loops with Quantitative Tube Formation Image Analysis software (WimTube, Wimasis; https://www.wimasis.com/en/products/13/WimTube).

To further clarify the MAPK signaling pathway in the angiogenic capacity of DPCs, cells were preconditioned with U0126, SB203580 and SP600125 $(10 \mu \mathrm{mol} / \mathrm{ml}$; Gene Operation) prior to addition of EPO and subsequent experiments were performed as described above.

Protein array analysis. The Human Cytokine Antibody Array Kit (RayBiotech, Inc) was used to identify angiogenesis-related factors within the CM of DPCs, which were treated and collected as described above. The assay was performed strictly according to the manufacturer's protocol. The chip was scanned with a GenePix 4000B Microarray Scanner (Molecular Devices, LLC) using $\mathrm{Cy} 3$ as the fluorophore. The binding signals were acquired and analyzed by applying the RayBio $^{\circledR}$ software tool (AAH-CUST-G; RayBiotech, Inc).

ELISA. To confirm the results of the protein array using quantitative analysis, the protein concentrations of MMP-3 (cat. no. DMP300), angiopoietin-1 (Ang-1) (cat. no. DY923), IL-6 (cat. no. D6050) and TGF- $\beta$ (cat. no. DB100B) were detected using ELISA kits (R\&D Systems, Inc.) according to the manufacturer's protocol. ELISAs were performed on the CM of DPCs treated with different concentrations of EPO.

Western blot analysis. DPCs were preconditioned with $40 \mathrm{U} / \mathrm{ml}$ EPO for $1 \mathrm{~h}$ and lysed in RIPA buffer containing proteinase inhibitor cocktail (Beyotime Institute of Biotechnology). The protein was quantified using a BCA Protein Assay Kit. Western blot analysis was performed as instructed by the manufacturer using a capillary-based automated system WES (Protein Simple) which uses capillary electrophoresis to identify and quantify a protein of interest. In brief, protein samples of $0.5 \mathrm{mg} / \mathrm{ml} / \mathrm{lane}$ were loaded. Next, primary and secondary antibodies and luminal peroxide mix were dispensed into the assay plate per the manufacturer's protocol. Primary antibodies included rabbit anti-human ERK1/2 (cat. no. 4695), phospProtein Simplep-p38(cat. no. 4511) (1:100; R\&D Systems, Inc.), JNK (cat. no. 9252), p-JNK (cat. no. 9255) (1:100; Cell Signaling Technology, Inc.) and $\beta$-actin (cat. no. 31-1013-00, 1:1,000; EarthOx Life Sciences). The secondary antibodies were included in the Simple Wes Kit (cat. no. 042-205 and cat. no. 042-206; (Protein Simple). Analysis of detected proteins was performed with Compass Software (version: 2.6.7; Protein Simple).

Statistical analysis. The experiments were performed in triplicate unless otherwise stated and quantitative results are expressed as the mean \pm standard deviation. Data were analyzed using the SPSS software (version 13.0; SPSS, Inc.). One-way ANOVA was performed for multiple-group comparisons, with post-hoc Bonferroni correction. $\mathrm{P}<0.05$ was considered to indicate a statistically significant difference. 
A

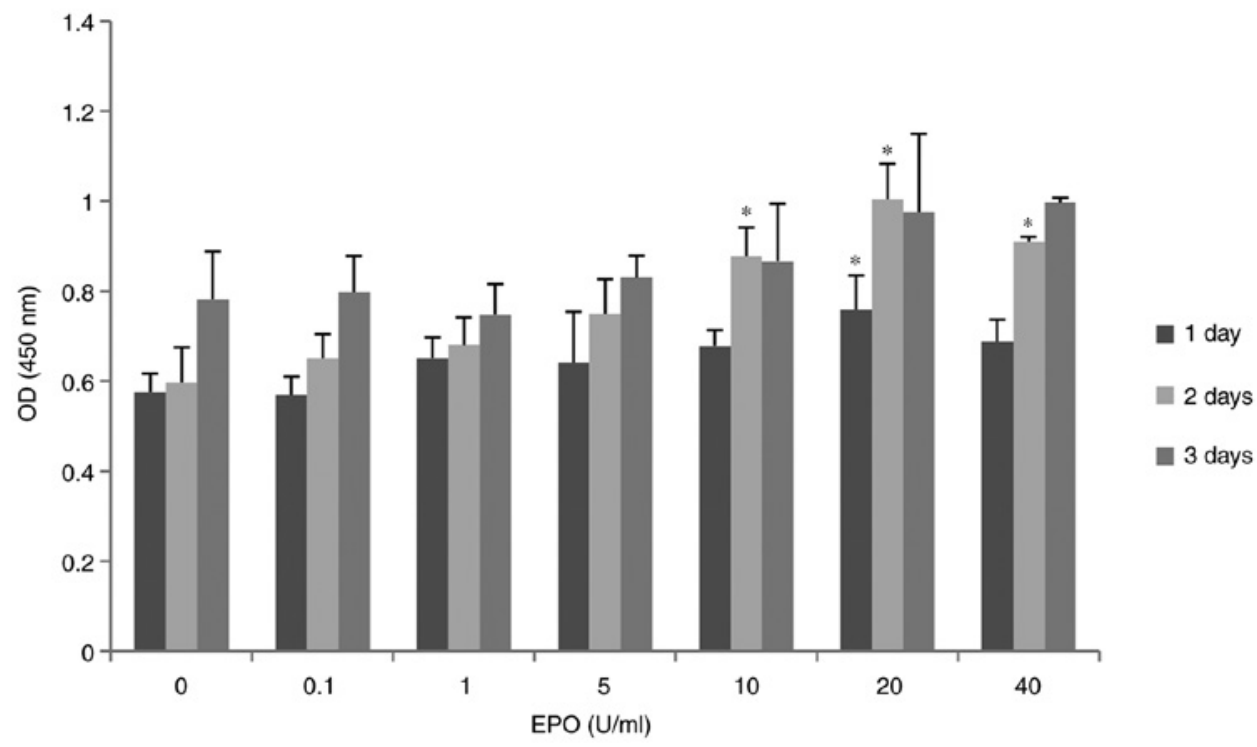

B
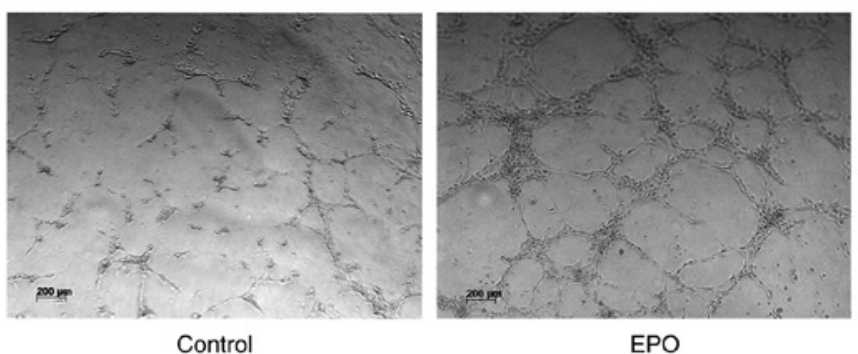

EPO
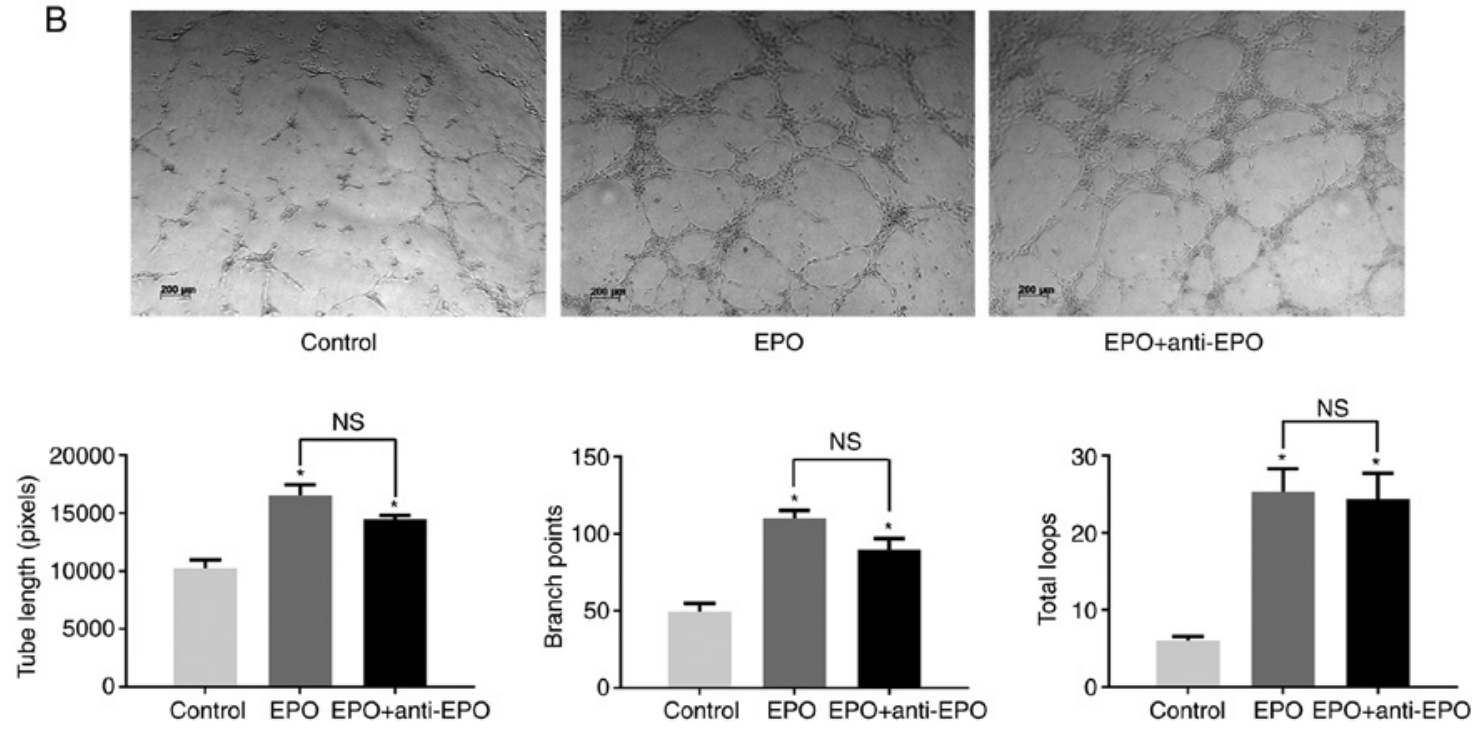

Figure 1. Effects of EPO on proliferation and angiogenesis of DPCs. (A) Cell Counting Kit-8 assay for the proliferation of DPCs cultured with different concentrations of EPO. Results are presented as the viability relative to untreated cells $(0 \mathrm{U} / \mathrm{ml} \mathrm{EPO})$ and values are expressed as the mean \pm standard deviation of three independent experiments. ${ }^{*} \mathrm{P}<0.05$ vs. untreated cells ( $\left.0 \mathrm{U} / \mathrm{ml} \mathrm{EPO}\right)$. (B) Matrigel tube formation assay of human umbilical vein endothelial cells in the conditioned medium of DPCs induced by EPO, with or without neutralization antibody (scale bar, $200 \mu \mathrm{m}$; magnification, $\mathrm{x} 50$ ) and quantitative results. Quantification was performed on 3 randomly selected fields. Values are expressed as the mean \pm standard deviation of three independent experiments. ${ }^{*} \mathrm{P}<0.05$ vs. Control group; NS, not significant. EPO, erythropoietin; DPCs, dental pulp cells; OD, optical density.

\section{Results}

Effects of EPO on the proliferation and angiogenesis of $D P C s$. The effect of EPO on the proliferation of DPCs was assessed with a CCK-8 assay. As presented in Fig. 1A, low concentrations of EPO $(0.1,1$ or $5 \mathrm{U} / \mathrm{ml})$ had no effect on DPC proliferation at any time-point. However, the proliferation of DPCs was significantly above that of the control $(\mathrm{P}<0.05)$ when exposed to 10, 20 or $40 \mathrm{U} / \mathrm{ml}$ EPO for 2 days. These results indicated that high concentrations of EPO promoted the proliferation of DPCs.

In the Matrigel assay, vessel-like structures of HUVECs were observed in the EPO group. By contrast, HUVECs in the control group assembled into vessel wall analogs but hardly formed any loops. Furthermore, addition of EPO neutralization antibody did not significantly attenuate tube formation compared with the EPO group. Quantitative data indicated that the tube lengths, branch points and total loop amounts significantly increased in the EPO group and EPO with antibody group $(\mathrm{P}<0.05)$. In addition, no significant differences were observed between the EPO group and EPO group with antibodies (Fig. 1B).

Expression of various angiogenesis-related factors in CM of DPCs stimulated by EPO. The relative expression of 35 angiogenesis-related factors, including 29 pro-angiogenic cytokines and 6 anti-angiogenic factors in the CM of DPCs, was detected by the human angiogenesis protein array. As indicated in Fig. 2A-D and Table I, the expression of vascular endothelial cell growth factor (VEGF), MMP-3, Ang-1/2 and hepatocyte growth factor (HGF) significantly increased by $>50 \%$, while the secretion of TGF- $\beta$, TNF- $\alpha$, IL-2, IL-6 
A

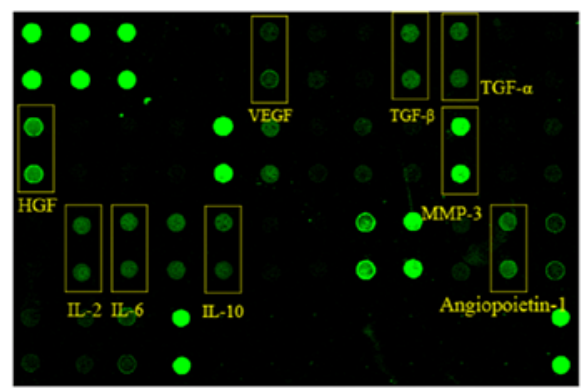

C

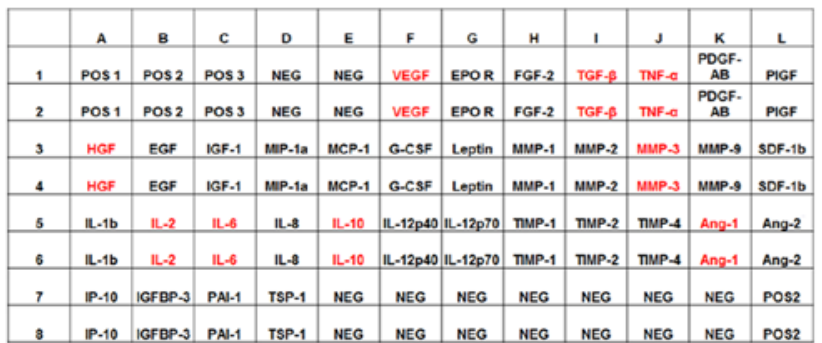

B

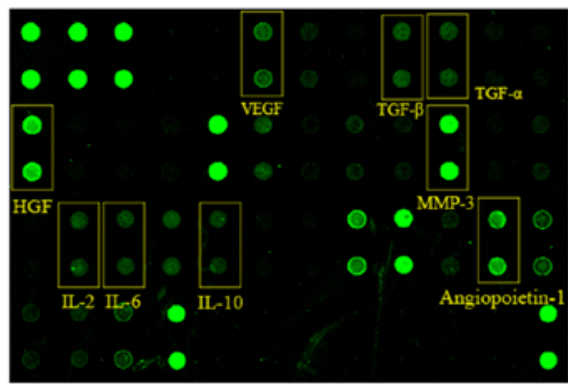

D

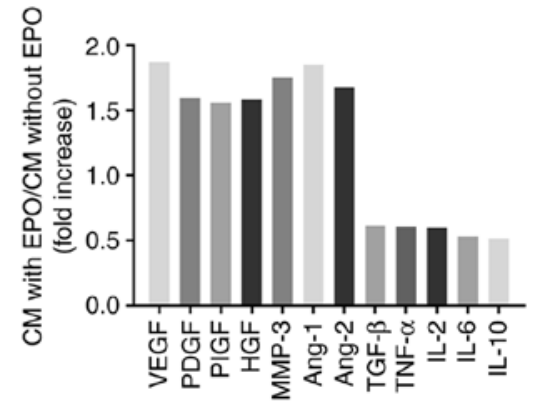

E
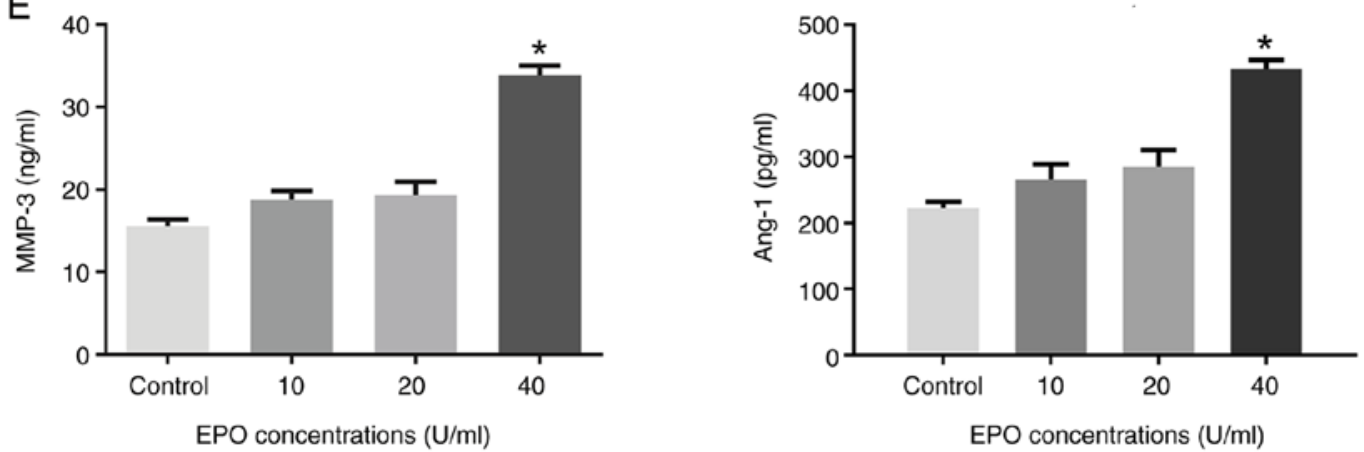

EPO concentrations $(\mathrm{U} / \mathrm{ml})$
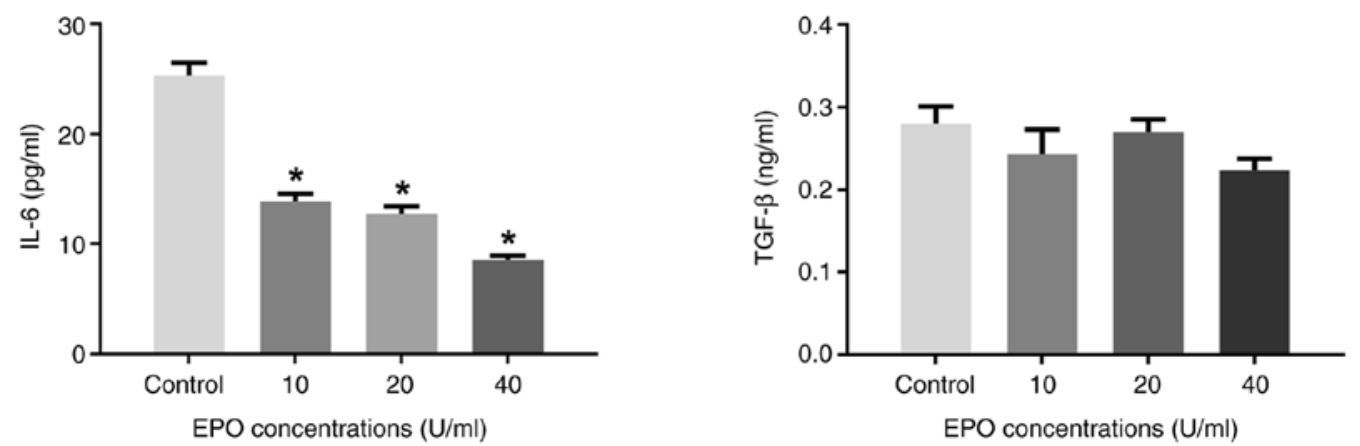

Figure 2. Protein array and ELISA of CM of DPCs. (A) Protein array image of CM from DPCs; (B) protein array image of CM from DPCs treated with EPO (C) Map of the protein array; positive proteins are marked in red. (D) Fold changes of the fluorescence signals of positive proteins. (E) ELISA was used to determine angiogenesis-associated cytokines MMP3, Ang-1, IL-6 and TGF- $\beta$ in the CM of DPCs treated with graded concentrations of EPO. Values are expressed as the mean \pm standard deviation of three independent experiments. ${ }^{*} \mathrm{P}<0.05$ vs. Control group. EPO, erythropoietin; DPCs, dental pulp cells; CM, conditioned medium; NEG, negative control; POS, positive control; VEGF, vascular endothelial growth factor; PDGF, platelet-derived growth factor; HGF, hepatocyte growth factor; Ang-1, angiopoietin-1; PIGF, phosphatidylinositol glycan anchor biosynthesis class F; IGF, insulin-like growth factor; IGFBP, IGF binding protein; EGF, epidermal growth factor; MIF, macrophage migration inhibitory factor; MCF, monocyte chemoattractant protein; G-CSF, granulocyte colony-stimulating factor; FGF, fibroblast growth factor; TIMP1, TIMP metallopeptidase inhibitor 1; SDF, stromal cell-derived factor; PAI-1, plasminogen activator inhibitor- 1; IP-10,interferon inducible protein-10; MIP-1a, macrophage inflammatory protein-1a; TSP, thrombin-sensitivity protein.

and IL-10 decreased to $40-50 \%$ compared with CM from DPCs untreated with EPO. In addition, the expression of 4 out of 6 anti-angiogenic factors (IL-10, IL-12p70, TIMP-1 and TIMP-4) decreased in the CM of DPCs after EPO stimulation.

Furthermore, ELISA indicated that $40 \mathrm{U} / \mathrm{ml}$ EPO significantly increased the secretion of MMP-3 and Ang-1 in comparison with the control group $(\mathrm{P}<0.05)$. However, lower concentrations (10 and $20 \mathrm{U} / \mathrm{ml}$ ) of EPO had no significant effect on MMP-3 and Ang-1 production in DPCs. Furthermore, EPO significantly decreased the secretion of IL-6 but had no obvious effect on TGF- $\beta$ secretion (Fig. $2 \mathrm{E}$ ).

Intracellular pathways of the DPCs activated by EPO. For evaluation of the activity of MAPK, the ratio of phosphorylated 
Table I. Profile of cytokines in conditioned medium of dental pulp cells induced by EPO.

A, Pro-angiogenic factors and receptors

\begin{tabular}{lc}
\hline Cytokine & EPO/control ratio \\
\hline VEGF & 1.871 \\
EPOR & 1.268 \\
bFGF & 0.849 \\
TGF- $\beta$ & 0.616 \\
TNF- $\alpha$ & 0.609 \\
PDGF-AB & 1.595 \\
PIGF & 1.557 \\
HGF & 1.585 \\
EGF & 1.299 \\
IGF-1 & 0.777 \\
Ang-1 & 1.849 \\
Ang-2 & 1.677 \\
IGFBP-3 & 1.200 \\
Leptin & 0.931 \\
MMP-1 & 0.834 \\
MMP-2 & 1.078 \\
MMP-3 & 1.750 \\
MMP-9 & 1.302 \\
IL-1 $\beta$ & 1.241 \\
IL-2 & 0.597 \\
IL-6 & 0.531 \\
IL-8 & 0.705 \\
PAI-1 $\beta$ & 1.001 \\
IL-12p40 & 0.896 \\
MIP-1 $\alpha$ & 0.930 \\
MCP-1 & 0.950 \\
IP-10 & 0.858 \\
\hline
\end{tabular}

B, Anti-angiogenic cytokines

\begin{tabular}{lc}
\hline Cytokine & EPO/control ratio \\
\hline IL-10 & 0.518 \\
IL-12p70 & 0.950 \\
TIMP-1 & 0.687 \\
TIMP-2 & 1.272 \\
TIMP-4 & 0.898 \\
Thrombospondin-1 & 1.403 \\
\hline
\end{tabular}

VEGF, vascular endothelial growth factor; PDGF, platelet-derived growth factor; EPO, erythropoietin; HGF, hepatocyte growth factor; Ang-1, angiopoietin-1; PIGF, phosphatidylinositol glycan anchor biosynthesis class F; IGF, insulin-like growth factor; IGFBP, IGF binding protein; EGF, epidermal growth factor; MIF, macrophage migration inhibitory factor; $\mathrm{MCF}$, monocyte chemoattractant protein; G-CSF, granulocyte colony-stimulating factor; bFGF, bone-derived fibroblast growth factor; TIMP1, TIMP metallopeptidase inhibitor 1; SDF, stromal cell-derived factor; PAI-1, plasminogen activator inhibitor- 1; IP-10,interferon inducible protein-10; MIP-1a, macrophage inflammatory protein-1a; TSP, thrombin-sensitivity protein.

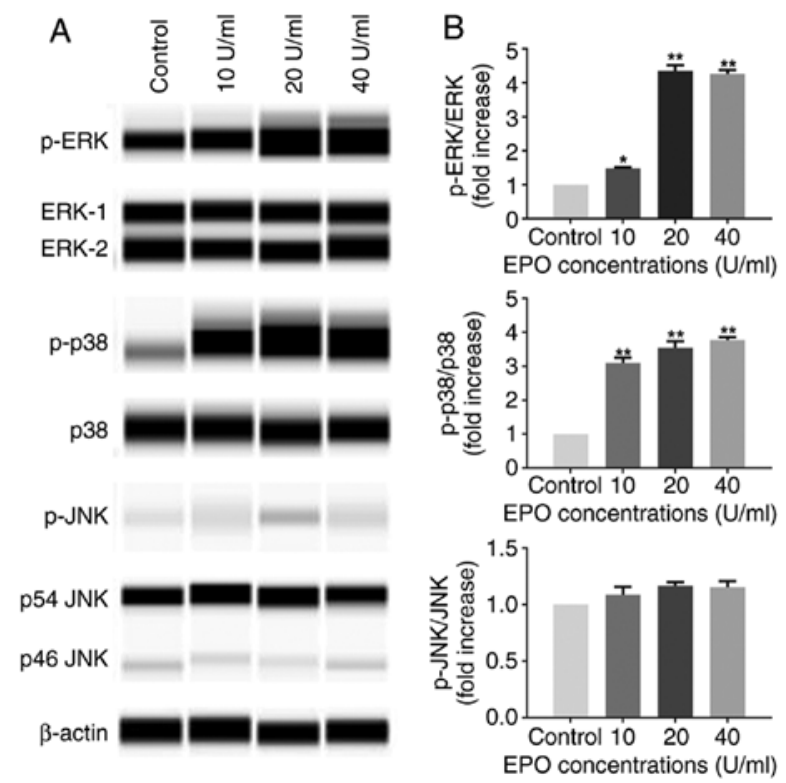

Figure 3. Related intracellular pathways of the DPCs activated by EPO. (A) Western blot bands for various proteins of the MAPK signaling pathway in DPCs cultured with different concentrations of EPO. (B) Quantitative analysis of p-ERK/ERK, p-p38/p38 and p-JNK/JNK in DPCs induced by EPO. Values are expressed as the mean \pm standard deviation of three independent experiments. ${ }^{*} \mathrm{P}<0.05 ;^{* *} \mathrm{P}<0.01$ vs. Control group. EPO, erythropoietin; DPCs, dental pulp cells; p-JNK, phosphorylated JNK.

to total protein was quantified. As indicated in Fig. 3, the levels of p-ERK and p-p38 were significantly increased in DPCs after exposure to EPO for $1 \mathrm{~h}$, whereas the phosphorylation levels of JNK were not enhanced compared with the control group. However, the total protein levels of ERK1/2, p38 and JNK were maintained at the same level under EPO stimulation.

Signaling pathways involved in EPO-induced angiogenesis of DPCs. To further determine the molecular mechanisms of EPO-induced angiogenesis, DPCs were pretreated with MAPK signaling pathway inhibitors and then subjected to EPO stimulation. Subsequently, CM from DPCs was collected to stimulate HUVECs. As presented in Fig. 4, both U0126 (ERK1/2 inhibitor) and SB203580 (p38 inhibitor) impaired the formation of capillary-like structures, while SP600125 (JNK inhibitor) had no significant effect on tube formation, which was also confirmed by the quantitative data.

\section{Discussion}

Dental pulp regeneration is challenging due to the special structure of the enamel-dentin-pulp complex and the limited blood and oxygen supply. Numerous studies have focused on the key role of DPCs, as they have multidifferentiation potential and affect stem cells in a paracrine manner. EPO has been indicated to protect against hypoxia and ischemia and exert anti-inflammatory, anti-apoptosis and pro-angiogenesis effects within numerous organs (17-19). Koutsoumparis et al (20) indicated that recombinant human EPO promoted endothelial transdifferentiation of stem cells from the apical papilla, which may be of clinical value in the treatment of ischemic disorders. The present study was the first, to the best of our knowledge, to report that EPO promoted the angiogenic potential of DPCs in 

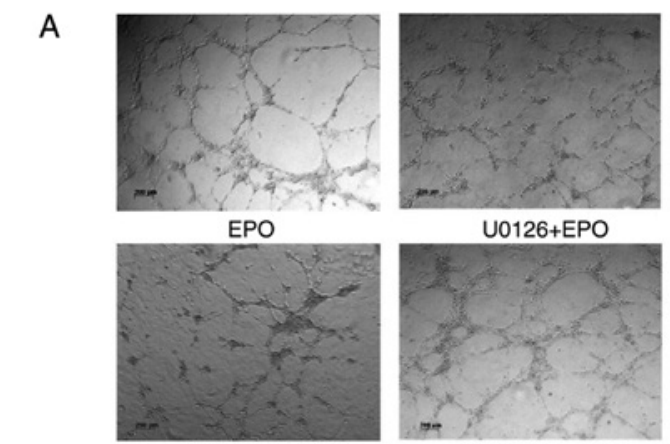

U0126+EPO
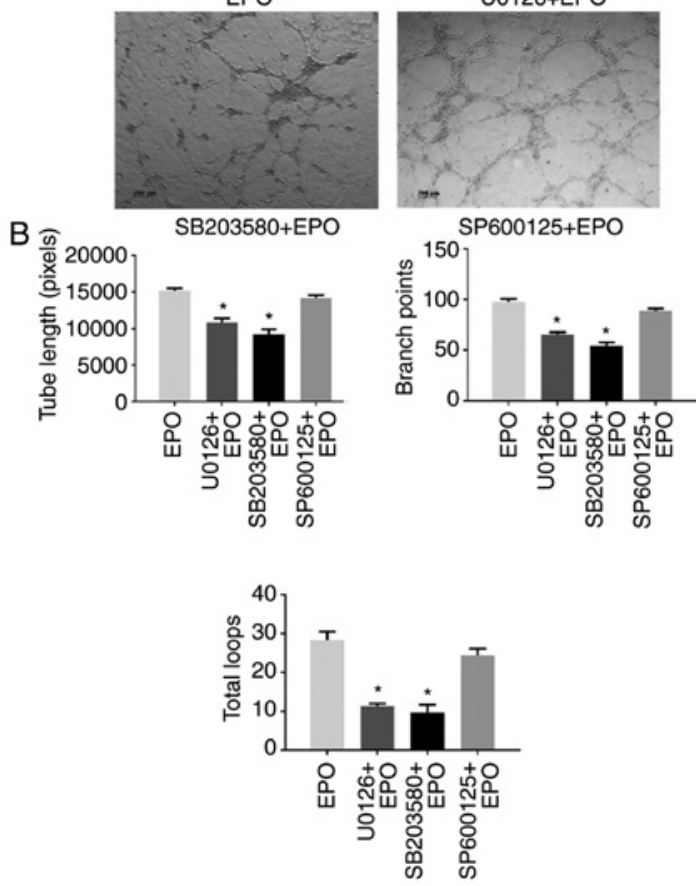

Figure 4. Effects of MAPK pathway inhibitors on the angiogenesis of DPCs induced by EPO. (A) Effects of U0126 (ERK1/2 inhibitor), SB203580 (p38 inhibitor) and SP600125 (JNK inhibitor) on the tube formation of human umbilical vein endothelial cells in the conditioned medium of EPO-induced DPCs by. (B) Quantitation of the Matrigel tube formation assay. Values are expressed as the mean \pm standard deviation of three independent experiments (scale bar, $200 \mu \mathrm{m}$; magnification, x 50). ${ }^{*} \mathrm{P}<0.05$ vs. EPO group. EPO, erythropoietin; DPCs, dental pulp cells.

a paracrine manner. EPO stimulated the secretion of various angiogenic cytokines in DPCs and thus promoted tube formation of endothelial cells through ERK1/2 and p38 MAPK signaling.

Proliferation and migration are the primary steps of dental pulp repair. Lin et al (21) demonstrated that EPO induced the proliferation of MSCs and overexpression of EPO enhanced the migration capacity of MSCs. Zwezdaryk et al (22) revealed that low levels of EPO promoted human MSC proliferation, whereas high levels of EPO ( $>30 \mathrm{U} / \mathrm{ml})$ more successfully affected cell migration and angiogenesis potential. Previous research by our group also indicated that EPO increased C-X-C motif chemokine receptor 4 and stromal cell-derived factor-1 expression and enhanced DPC migration in vitro (data not shown). Consistently, it was observed in the present study that the proliferation of DPCs increased the most in the $20 \mathrm{U} / \mathrm{ml}$ EPO group, while a lower concentration of EPO had no such effect. However, other studies reported that EPO had no effects on bone marrow stromal cell proliferation $(23,24)$. Therefore, it was speculated that the inconsistency of these studies was due to different types of EPO, cell types and experimental conditions; and EPO was potent only if its concentration reached a certain magnitude and sufficient EPOR requires to be activated to achieve biological efficiency.
Various studies confirmed that EPO promoted tissue repair by supporting or inducing angiogenesis $(25,26)$, but whether EPO has similar effects on DPCs has remained elusive. Previous studies have demonstrated that autocrine and/or paracrine factors are the secretion mechanism of MSCs within tissue regeneration (27). Likewise, different research groups performed studies on paracrine effects of DPSC-CM on endothelial cells and angiogenesis. Shen et al (28) reported that DPSC-derived CM significantly promoted angiogenesis in hind limb ischemia, suggesting that paracrine effects are of great importance in angiogenesis. In the present study, it was observed that CM from DPCs stimulated by EPO significantly promoted the formation of vessel-like structures by HUVECs. Addition of EPO antibody did not significantly attenuate angiogenesis, which not only excluded the direct effects of EPO but also demonstrated that EPO enhanced tube formation by HUVECs through the cytokines secreted by DPCs.

However, the molecular mechanisms by which EPO promotes angiogenesis through DPCs have remained to be fully elucidated. Thus, a protein array was performed in the present study to examine the differential expression profile of cytokines in CM of DPCs treated with EPO. Serum-free CM was used to simulate the inflammatory environment of injured pulp. Among all the angiogenesis-related factors selected, the secretion of pro-angiogenesis factors in CM, such as VEGF, HGF, MMP-3, Ang-1 and Ang-2, were all markedly upregulated, whereas TGF- $\beta$, TNF- $\alpha$, IL- 2 , IL- 6 and IL-10 were downregulated after EPO treatment. Since the downregulated cytokines are also pro-inflammatory cytokines, these changes may be speculated to be related to the possible anti-inflammatory effects of EPO (29-31). Subsequently, ELISA confirmed that MMP-3 and Ang-1 in the CM was upregulated in an EPO dose-dependent manner, while downregulation of IL-6 secretion was detected by the protein array. According to previous studies, MMP-3 and Ang-1 are vital factors involved in the process of angiogenesis in dental pulp and other tissues $(32,33)$. IL-6, as an inflammatory cytokine, has a critical role in dental pulp inflammation (34). These results suggested that EPO may exert angiogenic potential and anti-inflammatory activity in dental pulp repair. Xu et al (35) indicated that hydrogels loaded with aspirin/EPO are effective in the control of inflammation and regeneration of the periodontium. A recent study also demonstrated that an EPO-impregnated collagen scaffold promoted new bone formation in an alveolar defect through coupled angiogenesis/osteogenesis (36). Indeed, the mineralization of the dentin matrix during dentinogenesis is similar to the osteogenesis processes. Based on the present and previous results, it was hypothesized that engineered scaffolds loaded with EPO may open new avenues for future research in dental pulp regeneration and provide a promising strategy for clinical applications for the treatment of pulpitis.

Activation of EPOR usually triggers three major intracellular signaling cascades: JAK2/STAT5, PI3K/Akt and RAS/MAPK. The activation of MAPK pathways is known to respond to various exogenous stresses by ischemia and is involved in angiogenesis. Kwon et al (37) indicated that EPO induced the gradual elevation of ERK1/2 and p38 expression as time progressed in C6 glioma cells and the protective effects of EPO against cytotoxicity were significantly attenuated with pretreatment with p-ERK1/2 or p-p38 inhibitor. In the present study, the phosphorylation of ERK1/2, p38, and JNK was investigated and 
the results demonstrated that EPO increased the angiogenesis capacity of DPCs through ERK1/2 and p38 signaling but not JNK. However, contrary to the present results, $\mathrm{p}-\mathrm{JNK}$ protein levels decreased significantly in the EPO group in a rat model of ischemia-reperfusion injury (38). It may be inferred that the activation of signaling pathways may vary from species to species.

In conclusion, the present results revealed that EPO enhanced the angiogenic potential of DPCs through the secretion of angiogenic cytokines under serum deprivation conditions. In addition, ERK1/2 and p38 MAPK signaling pathways participate in this process. Although whether EPO is effective in vivo remains to be determined, these results indicated that EPO has an essential role in dental pulp repair and may be applied in pulp regeneration.

\section{Acknowledgements}

Not applicable.

\section{Funding}

This study was supported by the National Natural Science Foundation of China (grant nos. 81870750 and 81700957), the Natural Science Foundation of Guangdong Province (grant no. 2016A030310197) and the Fundamental Research Funds for the Central Universities (grant no. 12ykpy65).

\section{Availability of data and materials}

The datasets used and/or analyzed during the current study are available from the corresponding author on reasonable request.

\section{Authors' contributions}

JL conceived the present study. QG, JZ, YH, CC and JQ performed the experiments. QG, JZ and $\mathrm{XZ}$ designed the study, analyzed the data and wrote the manuscript. All authors read and approved the final manuscript. QG and JZ confirm the authenticity of the raw data.

\section{Ethics approval and consent to participate}

All experiments were approved by The Research Ethics Committee of Hospital of Stomatology, Guanghua School of Stomatology, Sun Yat-sen University.

\section{Patient consent for publication}

Not applicable.

\section{Competing interests}

The authors declare that they have no competing interests.

\section{References}

1. Saghiri MA, Asatourian A, Sorenson CM and Sheibani N: Role of angiogenesis in endodontics: contributions of stem cells and proangiogenic and antiangiogenic factors to dental pulp regeneration. J Endod 41: 797-803, 2015.

2. Liekens S, De Clercq E and Neyts J: Angiogenesis: Regulators and clinical applications. Biochem Pharmacol 61: 253-270, 2001
3. Liu L, Ling J, Wei X, Wu L and Xiao Y: Stem cell regulatory gene expression in human adult dental pulp and periodontal ligament cells undergoing odontogenic/osteogenic differentiation. J Endod 35: 1368-1376, 2009.

4. Bronckaers A, Hilkens P, Fanton Y, Struys T, Gervois P, Politis C, Martens W and Lambrichts I: Angiogenic properties of human dental pulp stem cells. PLoS One 8: e71104, 2013.

5. Paschalidis T, Bakopoulou A, Papa P, Leyhausen G, Geurtsen W and Koidis P: Dental pulp stem cells' secretome enhances pulp repair processes and compensates TEGDMA-induced cytotoxicity. Dent Mater 30: e405-e418, 2014.

6. Gharaei MA, Xue Y, Mustafa K, Lie SA and Fristad I: Human dental pulp stromal cell conditioned medium alters endothelial cell behavior. Stem Cell Res Ther 9: 69, 2018.

7. Kichenbrand C, Velot E, Menu P and Moby V: Dental pulp stem cell-derived conditioned medium: An attractive alternative for regenerative therapy. Tissue Eng Part B Rev 25: 78-88, 2019.

8. Tilbrook PA and Klinken SP: Erythropoietin and erythropoietin receptor. Growth Factors 17: 25-35, 1999.

9. Yan F, Zhang M, Meng Y, Li H, Yu L, Fu X, Tang Y and Jiang C: Erythropoietin improves hypoxic-ischemic encephalopathy in neonatal rats after short-term anoxia by enhancing angiogenesis. Brain Res 1651: 104-113, 2016.

10. Park SL, Won SY, Song JH, Kambe T, Nagao M, Kim WJ and Moon SK: EPO gene expression promotes proliferation, migration and invasion via the p38MAPK/AP-1/MMP-9 pathway by p21WAF1 expression in vascular smooth muscle cells. Cell Signal 27: 470-478, 2015.

11. Lu H, Wu X, Wang Z, Li L, Chen W, Yang M, Huo D, Zeng W and Zhu C: Erythropoietin-activated mesenchymal stem cells promote healing ulcers by improving microenvironment. J Surg Res 205: 464-473, 2016.

12. Zhu L, Bai X, Wang S, Hu Y, Wang T, Qian L and Jiang L: Recombinant human erythropoietin augments angiogenic responses in a neonatal rat model of cerebral unilateral hypoxia-ischemia. Neonatology 106: 143-148, 2014.

13. Wan L, Zhang F, He Q, Tsang WP, Lu L, Li Q, Wu Z, Qiu G, Zhou G and Wan C: EPO promotes bone repair through enhanced cartilaginous callus formation and angiogenesis. PLoS One 9: e102010, 2014.

14. Irrera N, Bitto A, Pizzino G, Vaccaro M, Squadrito F, Galeano M, Stagno d'Alcontres F, Stagno d'Alcontres F, Buemi M, Minutoli L, et al: Epoetin alpha and epoetin zeta: A comparative study on stimulation of angiogenesis and wound repair in an experimental model of burn injury. Biomed Res Int 2015: 968927, 2015.

15. Gong Q, Jiang H, Wei X, Ling J and Wang J: Expression of erythropoietin and erythropoietin receptor in human dental pulp. J Endod 36: 1972-1977, 2010.

16. Ammarguellat F, Llovera M, Kelly PA, Goffin V: Low doses of EPO activate MAP kinases but not JAK2-STAT5 in rat vascular smooth muscle cells. Biochem Biophys Res Commun 284: 1031-1038, 2001.

17. Suresh S, Rajvanshi PK and Noguchi CT: The many facets of erythropoietin physiologic and metabolic response. Front Physiol 10: 1534, 2020.

18. Hu R, Cheng Y, Jing $\mathrm{H}$ and $\mathrm{Wu} \mathrm{H}$ : Erythropoietin promotes the protective properties of transplanted endothelial progenitor cells against acute lung injury via PI3K/Akt pathway. Shock 42: 327-336, 2014.

19. Weng S, Zhu X, Jin Y, Wang T and Huang H: Protective effect of erythropoietin on myocardial infarction in rats by inhibition of caspase-12 expression. Exp Ther Med 2: 833-836, 2011.

20. Koutsoumparis A, Vassili A, Bakopoulou A, Ziouta A and Tsiftsoglou AS: Erythropoietin (rhEPOa) promotes endothelial transdifferentiation of stem cells of the apical papilla (SCAP). Arch Oral Biol 96: 96-103, 2018.

21. Lin H, Luo X, Jin B, Shi H and Gong H: The effect of EPO gene overexpression on proliferation and migration of mouse bone marrow-derived mesenchymal stem cells. Cell Biochem Biophys 71: 1365-1372, 2015.

22. Zwezdaryk KJ, Coffelt SB, Figueroa YG, Liu J, Phinney DG, LaMarca HL, Florez L, Morris CB, Hoyle GW and Scandurro AB: Erythropoietin, a hypoxia-regulated factor, elicits a pro-angiogenic program in human mesenchymal stem cells. Exp Hematol 35: 640-652, 2007.

23. Zhang D, Zhang F, Zhang Y, Gao X, Li C, Ma W and Cao K: Erythropoietin enhances the angiogenic potency of autologous bone marrow stromal cells in a rat model of myocardial infarction. Cardiology 108: 228-236, 2007. 
24. Koh SH, Noh MY, Cho GW, Kim KS and Kim SH: Erythropoietin increases the motility of human bone marrow-multipotent stromal cells (hBM-MSCs) and enhances the production of neurotrophic factors from hBM-MSCs. Stem Cells Dev 18: 411-421, 2009.

25. Kimáková P, Solár P, Solárová Z, Komel R and Debeljak N: Erythropoietin and its angiogenic activity. Int J Mol Sci 18: 1519, 2017.

26. Mizukami T, Iso Y, Sato C, Sasai M, Spees JL, Toyoda M, Umezawa A, Miyazaki A and Suzuki H: Priming with erythropoietin enhances cell survival and angiogenic effect of mesenchymal stem cell implantation in rat limb ischemia. Regen Ther 4: 1-8, 2016.

27. Baraniak PR and McDevitt TC: Stem cell paracrine actions and tissue regeneration. Regen Med 5: 121-143, 2010.

28. Shen CY, Li L, Feng T, Li JR, Yu MX, Lu Q and Li H: Dental pulp stem cells derived conditioned medium promotes angiogenesis in hindlimb ischemia. Tissue Eng Regen Med 12: 59-68, 2015.

29. Kai-Ian W and Si Z: Pretreatment with erythropoietin attenuates intestinal ischemia reperfusion injury by further promoting PI3K/Akt signaling activation. Transplant Proc 47: 1639-1645, 2015

30. Liu QS, Cheng ZW, Xiong JG, Cheng S, He XF and Li XC Erythropoietin pretreatment exerts anti-inflammatory effects in hepatic ischemia/reperfusion-injured rats via suppression of the TLR2/NF- $\kappa$ B pathway. Transplant Proc 47: 283-289, 2015.

31. Cravedi P, Manrique J, Hanlon KE, Reid-Adam J, Brody J, Prathuangsuk P, Mehrotra A and Heeger PS: Immunosuppressive effects of erythropoietin on human alloreactive T cells. J Am Soc Nephrol 25: 2003-2015, 2014.

32. Zheng $\mathrm{L}$, Amano $\mathrm{K}$, Iohara $\mathrm{K}$, Ito $\mathrm{M}$, Imabayashi $\mathrm{K}$, Into $\mathrm{T}$, Matsushita K, Nakamura $\mathrm{H}$ and Nakashima M: Matrix metalloproteinase-3 accelerates wound healing following dental pulp injury. Am J Pathol 175: 1905-1914, 2009.
33. Koh GY: Orchestral actions of angiopoietin-1 in vascular regeneration. Trends Mol Med 19: 31-39, 2013.

34. Elsalhy M, Azizieh F and Raghupathy R: Cytokines as diagnostic markers of pulpal inflammation. Int Endod J 46: 573-580, 2013.

35. Xu X, Gu Z, Chen X, Shi C, Liu C, Liu M, Wang L, Sun M, Zhang K, Liu Q, et al: An injectable and thermosensitive hydrogel: Promoting periodontal regeneration by controlled-release of aspirin and erythropoietin. Acta Biomater 86: 235-246, 2019.

36. Pandya M, Saxon M, Bozanich J, Tillberg C, Luan X and Diekwisch TGH: The glycoprotein/cytokine erythropoietin promotes rapid alveolar ridge regeneration in vivo by promoting new bone extracellular matrix deposition in conjunction with coupled angiogenesis/osteogenesis. Int J Mol Sci 22: 2788, 2021.

37. Kwon MS, Kim MH, Kim SH, Park KD, Yoo SH, Oh IU, Pak S and Seo YJ: Erythropoietin exerts cell protective effect by activating PI3K/Akt and MAPK pathways in C6 cells. Neurol Res 36: 215-223, 2014.

38. Pappo O, Ben-Ari Z, Shevtsov E, Avlas O, Gassmann M, Ravid A, Cheporko Y and Hochhauser E: The role of excessive versus acute administration of erythropoietin in attenuating hepatic ischemia-reperfusion injury. Can J Physiol Pharmacol 88: 1130-1137, 2010.

(i) $($ ) This work is licensed under a Creative Commons Attribution-NonCommercial-NoDerivatives 4.0 International (CC BY-NC-ND 4.0) License. 